\title{
Weightlessness alters up/down asymmetries in the perception of self-motion
}

\author{
Caty De Saedeleer • Manuel Vidal • Mark Lipshits • \\ Ana Bengoetxea • Ana Maria Cebolla • Alain Berthoz • \\ Guy Cheron · Joseph McIntyre
}

exiting each tunnel, subjects were asked to report their perception of the turn's angular magnitude by adjusting, with a trackball, the angular bend in a rod symbolizing the outside view of the tunnel. We demonstrate that the strong asymmetry between downward and upward pitch turns observed on Earth showed an immediate and signicant reduction when free-oating in weightlessness and a delayed reduction when the cosmonauts were rmly in contact with the oor of the station. These effects of weightlessness on the early processing stages (vestibular and optokinetics) that underlie the perception of self-motion did not stem from a change in alertness or any other uncontrolled factor in the ISS, as evidenced by the fact that weightlessness had no effect on the perception of yaw turns. That the effects on the perception of pitch may be partially overcome by haptic cues reects the fusion of multisensory cues and top-down inuences on visual perception.

Keywords Weightlessness · Asymmetry · Pitch · Perception

\section{Introduction}

The ability of humans to perceive and remember self-motion as they navigate through a $2 \mathrm{D}$ or $3 \mathrm{D}$ environment relies upon the integration of multimodal sensorimotor information, including static or dynamic visual cues, proprioception, vestibular cues, and corollary discharge (Mittelstaedt 1983,1999; Berthoz 1991, Glasauer and Mittelstaedt 1998; Vidal and Bülthoff 2009). It is reasonable to assume that these same sensory cues might contribute to the perception of what the physical environment offers in terms of potential motor actions (Sciutti et al. 2012). Addressing the question of how the CNS integrates spatial information from multiple 
sensory cues is fundamental to understanding the workings of the human brain (Pozzo et al. 1998).

In this study, we examined the role of gravitational information in the perception of self-motion in 3D. We used a paradigm in which a human subject observed visual stimuli corresponding to what they would see as they moved through a curved tunnel. The tunnel could turn in the horizontal plane to the right or to the left, or it could bend upward or downward in the sagittal plane. The task for the subject was to indicate the amplitude of the turn, based on the visual information provided. This task is of interest for the study of the macroscopic properties of sensorimotor integration because it requires a succession of processing: (1) temporal integration of the sensory input (spatial updating) during the transit through the tunnel, (2) working memory related to the perceived angle, and (3) retrieval (recall) of this angular information.

Previously, such a task revealed a signicant asymme try in pitch-induced perception on Earth. Downward stimuli produced a stronger pitch perception than upward, while leftward and rightward yaw turns were perceived equally (Vidal et al. 2006). This up-down asymmetry was also observed when subjects observed a static image of the tunnel (Vidal et al. 2006) and is similar to asymmetrical estimates of the slope of a hillside viewed either from above or from below (Proftt et al. 1995). To address the question of what reference frames are used to carry out the task, Vidal et al. (2006) recorded responses from human subjects who performed the tasks on Earth in either an upright, seated posture or while lying on their side. In doing so, the investigators decoupled the local, egocentric reference frame dened by the subject's body from the external, Earth-xed reference frame dened by gravity and somatosensory cues from the environment. They showed that (1) the up-down pitch asymmetry could arise in either reference frame, (2) asymmetries in OKN responses and the orientation with respect to gravity interacted to determine the response, and (3) the affordances offered by the visual scene could also interact with the sensory cues themselves to determine the perceived angle, depending on the orientation of the body with respect to gravity.

In the experiments reported here, we extended this study by testing the effects of gravity, or lack thereof, on the perception of the angle of the turn. We asked cosmonaut subjects to perform the experiment before, during and after long-duration space ight, and we manipulated vestibular versus haptic cues by having subjects perform the task in weightlessness, either in a rigidly xed posture with respect to the space station or during free-oating. We addressed two specic questions with these experiments, that is, "Does the lack of gravity or graviceptor input alter the sensory processing underlying the perception of self-motion?" and, if so, "Is it the lack of sensory signal per se that causes the disruption, or are any modications to the perception brought about by the differing affordances and consequences of actions that occur in the unique conditions of weightlessness?"

\section{Methods}

Figure 1 illustrates the experimental task. Subjects looked straight ahead through a form-tting face mask and a cylin drical barrel frame at the screen of a laptop computer onto which the images of virtual movements were displayed. The screen was centered on the line of gaze at a distance of $\sim 30 \mathrm{~cm}$ from the eyes. The barrel had a diameter of $16.5 \mathrm{~cm}$, yielding a circular eld of view subtending $30^{\circ}$ in all direc tions. The form-tting mask, the barrel, and, if necessary, turning off the lights in the room prevented any external

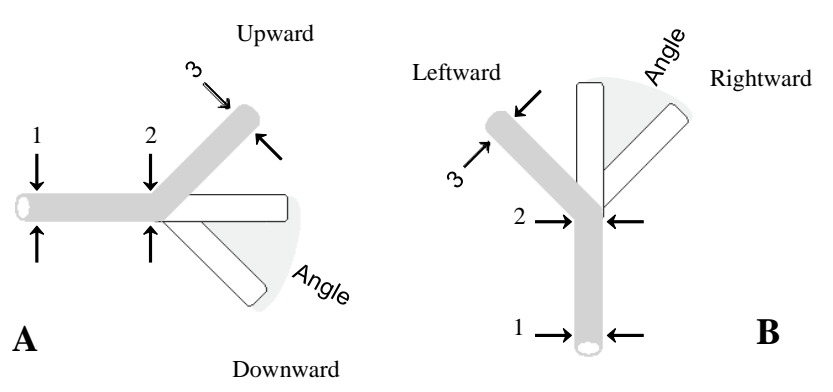

C

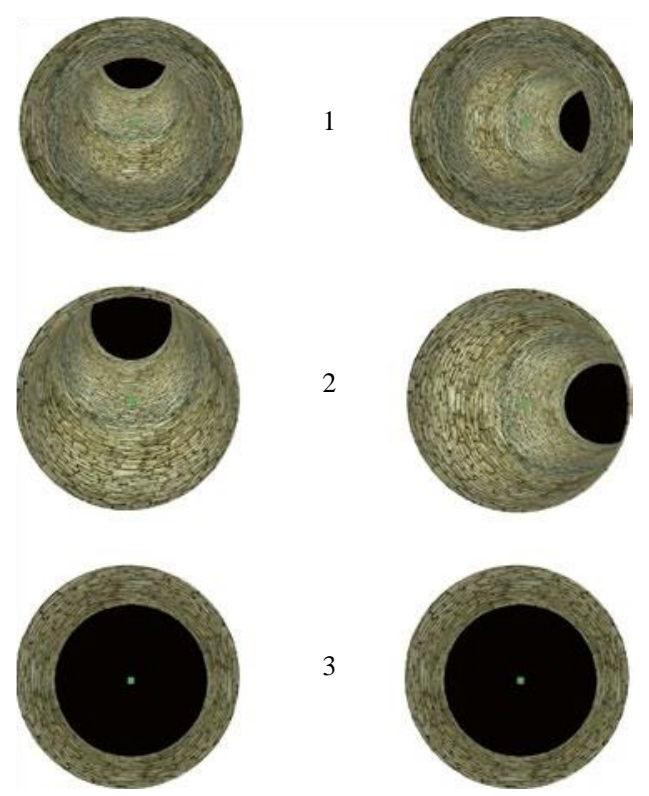

Fig. 1 Simulation of passive self-motion inside a tunnel with a bend in an upward or downward (a-c) and leftward or rightward (b-d) direction. a, b Response indicator in the form of a schematic outside view of the tube, viewed from the side for pitch (a) and from the top for yaw (b); c, d entry (1), bend (2), and exit (3) images for turn angle of $25^{\circ}$ for pitch (c) and for yaw (d) 
visual distractions and removed all external visual references (Cheron et al. 2006).

Subjects observed on the laptop screen a visual ow corresponding to simulation of passive self-motion inside tunnels in the form of a pipe of constant circular cross section, with stone-textured walls. Each tunnel contained an initial linear segment, a single turn in the middle, either in pitch or in yaw (Fig. 1), with one of 6 possible turn angles ranging from $25^{\circ}$ to $87.5^{\circ}$ in increments of $12.5^{\circ}$ and a nal linear segment. Subjects were driven passively at constant speed through the virtual 3D tunnel structure. Images were nonstereoscopic but included perspective cues generated by the OpenGL graphics libraries. Using a typical subject height of $1.75 \mathrm{~m}$ as a reference, the visual ow corresponded to a virtual speed of $2.21 \mathrm{~m} / \mathrm{s}$ (around $8 \mathrm{~km} / \mathrm{h}$ ), corresponding to a fast walking speed for humans. (Additional comments on the realism of the virtual motion can be found in Vidal et al. 2003). After exiting each tunnel, subjects were asked to report their perception of the turn's angular magnitude by adjusting a response indicator depicting an outside view of a tube on the laptop screen that could be bent by manipulating a trackball. The tube was viewed from the side for pitch turns and from the top for yaw turns (Fig. 1a, b). The tube was initially presented at $0^{\circ}$ (corresponding to a straight tunnel), and the subjects were instructed to bend the tube to the perceived turn amplitude by rolling the trackball. They pressed a button to indicate when they had reproduced the deviation angle corresponding to the angle perceived during the simulated movement. After a pause of $5 \mathrm{~s}$, subjects could initiate the subsequent trial with the push of a button.

An experimental session consisted of 48 trials, divided into four uninterrupted blocks of 12 trials. A given block included either exclusively pitch turns or exclusively yaw turns. All subjects began with a block of pitch turns, and then alternated, for a block sequence of pitch-yaw-pitchyaw. Each of the 6 possible amplitudes and two possible directions (leftward and rightward for yaw and upward or downward for pitch) occurred just once and in a random order in each block. At the end of a block, feedback about the subject's performance was displayed before a short pause. This feedback was the error (in degrees) measured between the real turn angle and the reported response, averaged over all trials in the block. Through this score, subjects were made aware of overall performance but received no information about what specic errors were committed. The experiment was preceded by four practice trials: two trials with pitch turns and two trials with yaw turns. During these trials, subjects learned how to use the computer interface, but received no feedback about performance. The full experiment lasted approximately $50 \mathrm{~min}$ for a complete session, which included the instructions and practice trials.

On Earth, subjects performed the experiment while sitting on a chair of adjustable height facing the computer/ barrel/mask that was placed on a table. During space ight, cosmonauts performed the experiment in two conditions. In the attached condition, cosmonauts used belts, foot straps, and a tabletop to maintain a sitting like posture in front of the laptop, like the one used on Earth. In the free-oating condition, subjects held the experimental apparatus (laptop computer and tunnel) in their hands with an elastic band holding the mask against the face. An assisting cosmonaut then positioned the subject in the center of the free working volume within one of the space station modules. The subject was released, and both subject and apparatus oated free from any contact with the station. The assisting cosmonaut ensured that no contact with the walls of the station occurred. To accomplish this, the assistant applied short tugs on the clothing of the subject to adjust the position trying to avoid giving strong directional cues. Very few such corrections (1-2 per session per subject) were required.

Figure 2 shows the testing schedule for the cosmonauts. Each cosmonaut was tested on 5 successive periods. Prior to ight, cosmonauts were tested on Earth in 2 pairs of sessions over the 2 months preceding liftoff (BF1, BF2). The two sessions within each period were separated by at least 1 day. These subjects were then tested on 2 days over the course of their spaceight aboard the ISS, with at least 1 day between sessions. Additionally, subjects performed the experiment twice on each day of testing on orbit, once in each of the two different experimental conditions ("attached" (A) and "freeoating" (FF)). The order of passage for the two postural conditions was counterbalanced across subjects. After their return from ISS, cosmonauts were tested on Earth again, on two different days during the week immediately following the landing (PF1) and two more times 1-3 weeks later (PF2).

Seven male cosmonauts $(\mathrm{C} 1-\mathrm{C} 7)$ participated in this investigation. The mean age $( \pm \mathrm{SD})$ of the cosmonauts was $42 \pm 3$ years. Six cosmonauts had previous experience in space ight; one cosmonaut (C2) had no such experience. All cosmonauts were in excellent health, as regularly determined by a special space ight medical commission dur ing all periods of the investigation. Six out of 7 cosmonauts performed the two sessions within the rst week of arriv ing on orbit (not before ight day 2 and not after ight day 7 ), and the seventh performed the experiment on ight days 16 and 18. The duration of exposure to weightlessness varied between subjects; four of whom spent 10 days on orbit (Russian-Belgian (ODISSEA) and Russian-Spanish (CERVANTES) "taxi" missions), while the other 3 cosmonauts spent 6 months aboard the ISS (Increments 9, 10, and 11).

Ten naive subjects ( 6 men and 4 women), $34 \pm 8$ years also participated in this experiment; most were students or laboratory staff, and all were right handed. These control subjects performed experimental sessions on the ground following the same schedule as cosmonauts. All participants gave prior written consent before starting this investigation. 


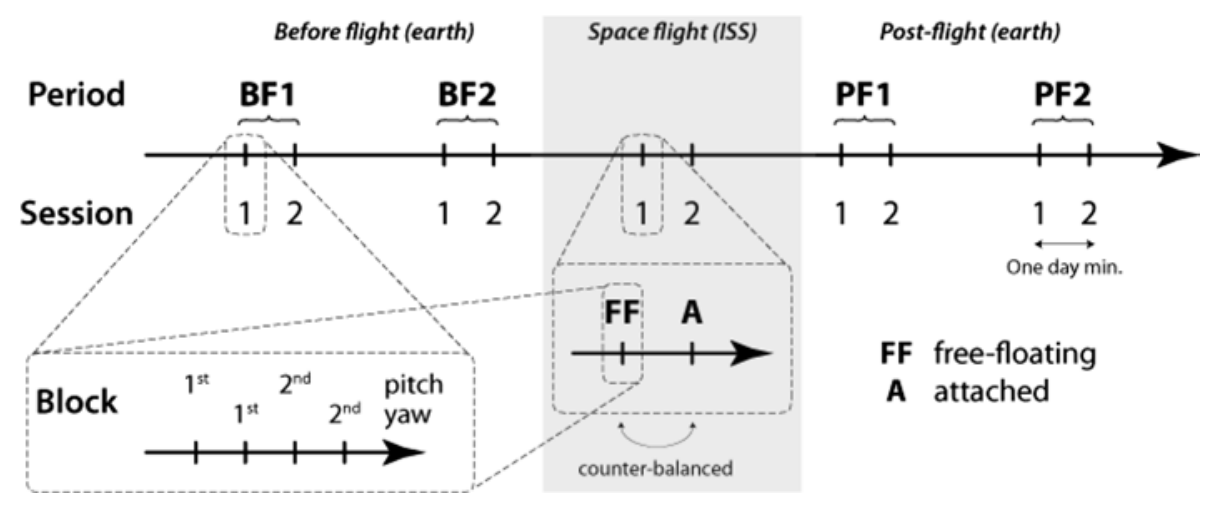

Fig. 2 Timeline of testing performed by each cosmonaut. The experiment was performed at different periods: 2 before, 1 during and 2 after ight aboard the ISS. Within each period, subjects were tested on two separate days, with at least 1 day in between. On each test day, subjects performed a total of 48 trials separated into 4 blocks of 12 trials each. Within each block, trials were all in pitch (up or down) or in yaw (left or right), and the order of trials was the same for each session (pitch-yaw-pitch-yaw). On each test day on board the ISS, subjects performed two sessions, one in an attached posture and one in free-oating. The order of these sessions on board the ISS was counterbalanced across subjects, with 3 performing attached rst and 4 performing free-oating rst

\section{Data analysis}

The perceived turn angle reported by subjects, and the response latencies (corresponding to the time that elapsed between the initial presentation of the response indicator and the moment that the subjects pressed the button to record the response) were recorded for each trial (48 trials/ session), distributed over 4 blocks of 12 trials each. We analyzed primarily the relative angular error as the difference between the real angle and the subject's response divided by the real angle (see Vidal et al. 2006), signed according to the following convention: positive if the reproduced angle was overestimated (overshot) and negative if it was underestimated (undershot).

Analysis of variance (ANOVA) with repeated measures was used to test for statistical signicance of the observed response errors. First, we applied separate ANOVA to pitch and yaw data for control subjects and for cosmonauts from their rst session on the ground, with direction (up vs. down for pitch, left vs. right for yaw) and turn magnitude $\left(25^{\circ}\right.$, $37.5^{\circ}, 50^{\circ}, 62.5^{\circ}, 75^{\circ}$, or $87.5^{\circ}$ ) as within-subject factors. Then, based on an observed main effect of turn direction for pitch, but not for yaw turns (see Results), and on previous studies (Vidal et al. 2006), we conducted specic planned comparisons on the computed "up/down asymmetry index" as the average error for pitch downward minus the average error for pitch upward stimuli, as a function of the real turn magnitude and as a function of the experimental session for each subject. Results from pre-ight tests on the ground showed that the up/down asymmetry was limited to the smallest stimulus angles $\left(25^{\circ}, 37.5^{\circ}, 50^{\circ}\right)$. We therefore dened the "small-angle up/down asymmetry" as the up/down asymmetry index averaged across these three angles. We tested whether any changes in perception could be directly attributed to the lack of gravity by comparing the small-angle up/down asymmetry for either free-oating or attached with the ground baselines. We also tested for a direct inuence of gravitational cues versus an indirect inu ence based on haptic cues by comparing the small-angle up/ down asymmetry for the attached versus free-oating pos ture on orbit.

\section{Results}

Figure 3 illustrates the relative angular error observed for the different real turn magnitudes in control subjects and cosmonauts. Control subjects who performed the experiment on the ground showed a marked asymmetry in the relative angular error for pitch (downward vs. upward) that was strongest for small angles and decreased progressively as the magnitude of the turn increased (Fig. 3a). A two-factor ANOVA revealed a signicant main effect of turn direction $\left(F_{1,9}=47.2, p<0.001\right)$ and of turn magnitude $\left(F_{5,45}=4.03\right.$, $p<0.01)$ and a signicant cross effect between these two factors $\left(F_{5,45}=17.8, p<0.0001\right)$. Scheffé's post hoc analysis showed that the difference between upward and downward response errors was signicant $(p<0.01)$ for the smaller magnitudes $\left(25^{\circ}, 37.5^{\circ}, 50^{\circ}\right)$, but not for the larger magnitudes $(p>0.20)$. We assessed the stability of these perceptual measures across repeated sessions by computing a two-way ANOVA on the up/down asymmetry index from the control subjects, with turn magnitude (six levels) and experiment period (ve levels) as within-subject fac tors. We found a signicant main effect of turn magnitude $\left(F_{5,40}=13.631, p<0.001\right)$, but no main effect of experiment 


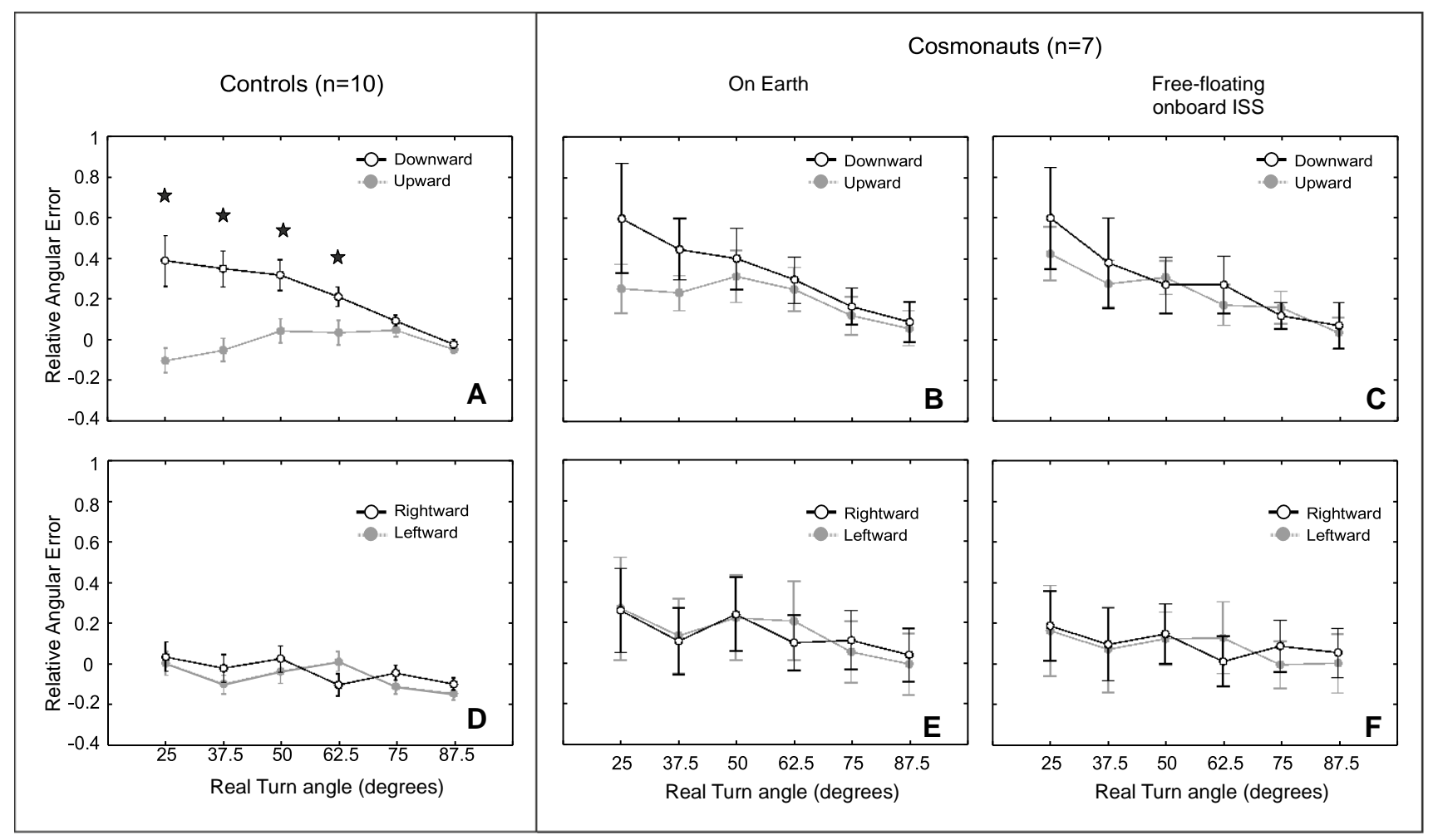

Fig. 3 The relative angular errors of perceived pitch $(\mathbf{a}-\mathbf{c})$ and yaw (d-f) turns for control subjects (a, d), cosmonauts on Earth before ight ( $\mathbf{b}, \mathbf{e})$, and cosmonauts free-oating in the ISS ( $\mathbf{c}, \mathbf{f})$. Gray line, lled circle : upward/leftward, Black line, open circle: downward/ rightward. Values are mean \pm SE. Stars indicate statistically signi cant $(p<0.05)$ differences between upward and downward as indicated by post hoc tests period and no cross effect. In other words, the pattern of up/ down asymmetry was stable over time for control subjects on the ground. The same control subjects on the ground showed no apparent differences between leftward and rightward bends (Fig. 3d), with no signicant main effects or interactions between the factors direction (left or right) and magnitude on relative angular error.

To test for effects of gravity on the perception of selfmotion in our virtual task, we analyzed the responses of cosmonaut subjects before, during and after their stay in the International Space Station. As a control, we rst assessed whether the conditions of spaceight altered the subjects' perception of the visual stimuli in a generic fashion, independent of a direct or indirect inuence of gravity on the updown asymmetries observed on Earth. We considered the perception of horizontal turns as baseline and compared the performance on these stimuli on Earth before the ight to weightlessness. As was the case for control subjects on the ground, a two-way ANOVA revealed no signicant effect of turn direction (left vs. right) or of turn magnitude $\left(25^{\circ}\right.$, $37.5^{\circ}, 50^{\circ}, 62.5^{\circ}, 75^{\circ}$ or $87.5^{\circ}$ ) for tests performed on the ground prior to the ight (Fig. 3e) while free-oating in weightlessness (Fig. 3f) or while in the attached condition in weightlessness (not shown). A one-way ANOVA comparison across these conditions (before ight, free-oat ing, attached) for leftward and rightward turns combined; showed no signicant effect of these experimental condi tions on relative angular error $\left(F_{2,12}=0.1024, p=0.90\right)$ even though response latencies were somewhat shorter, on average, for the two weightless conditions, compared to latencies of trials performed on the ground before ight ( $F_{2}$, $\left.{ }_{12}=4.73, p=0.0305\right)$. We can, therefore, conclude that cosmonaut's performance on the perceptual task was not affected by a change in alertness or any other uncontrolled factor during the sessions performed in weightlessness.

We then looked for a specic effect of gravity on per ceptual responses to pitch stimuli. Although somewhat less pronounced than for the control subjects, the cosmonauts manifested an up/down asymmetry in their responses on the ground prior to ight (Fig. 3b). A two-way ANOVA applied to the cosmonaut's responses to pitch stimuli on the ground showed a main effect of turn direction across all six turn magnitudes that was almost signicant $\left(F_{1,6}=5.4680\right.$, $p=0.05797$ ), and a planned comparison—justied by our previous observations on control subjects-showed that cosmonauts manifested a small-angle up/down asymmetry 
index signicantly different from zero $(p<0.002)$. While free-oating, the cosmonauts showed a qualitatively smaller difference between upward and downward turns (Fig. 3c). There was no main effect of turn direction $\left(F_{1,6}=0.72\right.$, $p=0.4267)$ nor any cross effect $\left(F_{5,30}=1.51, p=0.2137\right)$ in a two-way ANOVA (direction $\times$ magnitude) applied to pitch data, nor did the small-angle up/down asymmetry index differ signicantly from 0 ( $p=0.14)$. Interestingly, the suppression of the up/down asymmetry arose because the relative angular error increased for upward turns in weightlessness compared to ground, not because the error decreased for downward pitch.

To provide further statistical support for this observation, we looked for signicant changes in the asymmetry index across experimental sessions performed on ground and in weightlessness for the cosmonauts. To look for an immediate effect of gravity on perceptual responses, before any adaptation of task performance to the novel environment, we took the rst block of trials performed by each subject in each of the weightless conditions. We compared these data to the rst block of trials performed in each of the periods performed before and after ight, so that we compared tri als performed with a similar level of practice within each period. We considered trials performed in each period on the ground and the attached and free-oating postures on orbit as separate conditions, resulting in two-factor ANOVA with six levels for the factor "condition" (BF1, BF2, A, FF, PF1, PF2) and six levels for the factor "turn magnitude." With this analysis, we found the usual main effect of turn magnitude $\left(F_{5,30}=3.2028, p=0.01959\right)$ on the up/down asymmetry index, but we also found a signicant main effect of experiment condition $\left(F_{5,30^{\circ}}=2.7670, p=0.0359\right)$ and no cross effect.

The main effect of experimental conditions indicates that the lack of gravity could have had an effect on the perceptual asymmetry for pitch. This was conrmed by planned comparisons on the small-angle up/down asymmetry. Figure 4a shows the corresponding small-angle up/down asymmetry index before, during and after the space mission, for the rst block of trials performed in each condition. For
Fig. 4 Small-angle up/down asymmetry index (downward minus upward for turn angles $25^{\circ}, 37.5^{\circ}$, and $50^{\circ}$, combined) for the rst block ( a) and for the last block (b) of each experimental session. Values are averaged across 7 cosmonauts (mean \pm SE). Stars indicate statistically signicant $(p<0.05)$ differences from zero (lled stars) or between conditions (empty stars)
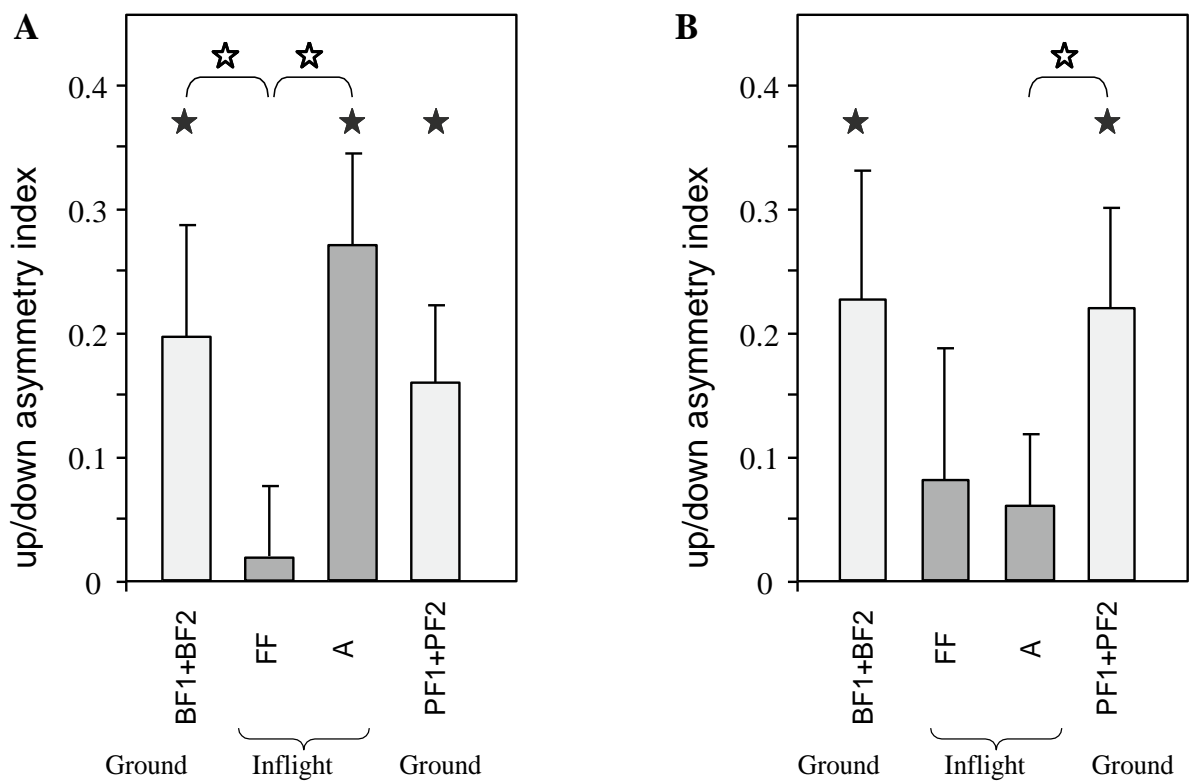

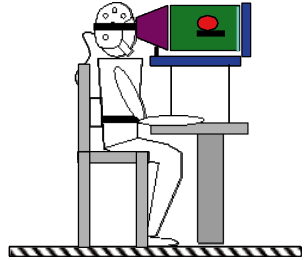

Ground

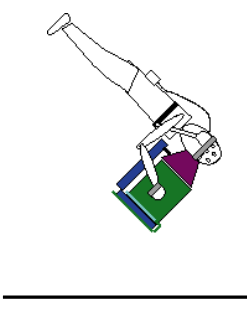

FF

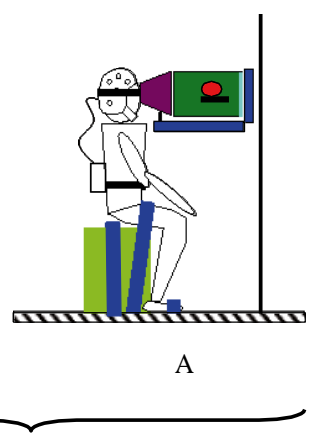

Inflight 
most conditions, the asymmetry index was signicantly dif ferent from zero for the small angles. The one exception is the trials performed in the free-oating condition on orbit. The small-angle asymmetry index for free-oating (mean index $=-1.76$ ) was not signicantly different from zero $(p=0.81)$ and was signicantly reduced ( $t$ test, $p<0.025)$

compared to its pre-ight value (mean index $=19.68$ ) . Interestingly, in the attached condition, the perceptual asymmetry for pitch was preserved in weightlessness: the small-angle up/down asymmetry index in this condition (mean index $=26.96$ ) was signicantly different from zero $(p=0.011)$ and did not differ from values before or after the ight. We also found that the small-angle up/down asymmetry differed signicantly between the attached and free-oating conditions in weightlessness ( $p<0.001)$. Note that similar statistical results for all these comparisons were obtained whether we considered data from just the rst block of trials performed during the rst day of testing in each condition, or whether we considered all trials (both blocks) performed on the rst day.

To test whether practice had any inuence on changes in perceptual asymmetries either on the ground or in weightlessness, we then compared the rst and last blocks of tri als performed within each testing period. Figure $4 \mathrm{~b}$ shows the small-angle up/down asymmetry for the second block of trials performed on the second day of testing within each period, that is, the most-practiced trials performed by each subject within each testing period and gravitational conditions. On the ground, practice had little or no effect on the up/down asymmetry, either before or after ight, that is, we found no statistical differences in the small-angle up/ down asymmetry between the rst block and last block of trials performed in each pre-ight and post-ight period. If anything, the average asymmetry index increased from the rst to the second day of testing in each of these ses sions, although this increase was not statistically signi cant. Similarly, the small-angle up/down asymmetry index did not change between the rst and last block of trials in the free-oating condition on orbit; in neither case was the small-angle up/down asymmetry index different from zero. Conversely, the asymmetry diminished with repetition for the attached condition in weightlessness. The small-angle up/down asymmetry index decreased signicantly between the rst (index $=29.6$ ) and last (index $=6.0$ ) block of trials performed in the attached condition in weightlessness $(p=0.013)$, such that it was no longer signicantly differ ent from zero ( $p=0.45$ ) or from the free-oating condition $(p=0.83)$ at the end of the second day of testing. Similar results were obtained when all trials on the second day of testing were compared to all trials from the rst. Thus, whereas the perceptual asymmetry for pitch was immediately diminished in free-oating and remained low through out the in-ight period, in the attached condition, the asymmetry disappeared only after a certain amount of time or practice in weightlessness (see "Discussion").

Finally, we note that in post-ight testing, the small-angle up/down asymmetry index was again statistically different from zero, both for the rst and for the last block of trials performed in each test period (or for the rst and last days combined), indicating the up/down asymmetry was restored on return to ground.

\section{Discussion}

In these experiments, we studied how gravitational information inuences the perception of scene geometry and the perception of self-motion based on visual cues. Three main results emerged from these psychophysical analyses. (1) The strong asymmetry between downward and upward pitch turns observed on Earth showed an immediate and signicant reduction once in weightlessness in the free-oating condition but not in the attached condition. (2) On the second test day of orbital ight, the perceptual asymmetry was also suppressed in the attached condition. (3) These effects do not stem from a change in alertness or any other uncontrolled factor during the ight sessions, as shown by the similar performance observed for yaw turns on ground and in ight. Taken together, these ndings provide evidence for two distinct mechanisms by which gravity (or the absence of) acts upon the perceptual asymmetry of pitch turns, depending on whether weightlessness is experienced within a stable somatosensory reference frame or not.

Global performance is conserved in weightlessness

The last of these results is in accordance with previous data (Vidal et al. 2003) showing that the accuracy to recreate an external image of the remembered 3D shape of the tunnel was not perturbed in weightlessness. Similarly, the ability to reproduce the orientation of a visual line was preserved during the same space missions (Lipshits et al. 2005). These results may at rst sight seem surprising in the context of visual sensory decits reported in early Soviet spacecraft mission (decrement in the ability to estimate the direction of line patterns, colors perception, and contrast sensitivity (Popov and Boyko 1967), alterations to the gaze holding system (Kornilova et al. 1983; Clément et al. 1993), and modications to receptor physiology during spaceight (Fuglesang et al. 2006). Difculties in reading check lists onboard the Shuttle have been reported and analyzed (NASA's EDOMP; Clément and Reschke 2008), but except for contrast sensitivity, no statistically signicant modica tions have been found for other parameters such as phoria, eye dominance, icker fusion frequency, and stereopsis. It should be noted, therefore, that our experiments were 
performed both on Earth and in the ISS in a fully controlled visual eld, protecting the subject from changes in visual environment (solar illumination and absence of atmospheric scattering of light). In these controlled conditions, visual perception per se does not appear to be substantially modied in weightlessness.

If not retinal physiology, then what?

It appears, then, that the processing of visual information originating in the retina is not the cause of either the perceptual asymmetry observed on Earth or its disappearance, given that neither anisotropy in the perception of visual orientation (Lipshits et al. 2005) nor basic physiological function of the retina (Task and Genco 1987) appear to be modied in weightlessness. What mechanisms might, there fore, underlie our observations that tilt perception is modied in space? As in the previous ground studies, both lowlevel reex processes and high-level cognitive function may come into play.

\section{Eye movements}

In our paradigm, the optokinetic system plays a central role and may partly explain the vertical anisotropy in favor of the downward pitch. Indeed, in experiments performed on the ground, suppressing eye movements reduced, but did not eliminate, the perceptual asymmetry between upward and downward turns (Vidal et al. 2006). On Earth, the gain of the optokinetic nystagmus (OKN) with slow phases directed upward (corresponding to downward pitch turns in our task) is larger than those corresponding to the OKN with slow phases directed downward and may thus contribute to the asymmetrical perception on Earth (Clément and Reschke 2008). It is also known that otolith stimulation greatly inu ences the vertical OKN (Igarashi et al. 1987; Clément and Lathan 1991; Gizzi et al. 1994). Interestingly, it was demonstrated that the vertical OKN asymmetry recorded in terrestrial conditions was reversed in weightlessness during the early period of the ight and showed a trend toward sym metry after 2 weeks in weightlessness (Clément et al. 1986, 1993; Clément 2003). This vertical OKN reversal might explain why upward pitch turns became overestimated as much as downward pitch turns, leading to the suppression of the up/down asymmetry in the free-oating condition.

In this context, the eye velocity of the OKN slow phase may be considered as an indicator of the effectiveness of image motion on the retina not only on the motor action of the eye but also on the nal perception. The otolith inputs play a regulating role in the vertical asymmetry by exerting an upward drive on eye movement (Clément and Reschke 2008). It was also reported by the inspection of the position of the nystagmus beating eld that the eye position of gaze was displaced upward during the early period of ight (Clément 2003), indicating a possible otolith-dependent change in eye position. This position signal is elaborated by a complex network of recurrent connections (the oculomotor neural integrator) performing the mathematical integration of the different velocity signals coming from vestibular, optokinetic, and pursuit system (Robinson 1989, Major et al. 2004). The vestibular-prepositus complex (Cheron et al. 1986a, b; Cheron and Godaux 1987) and the interstitial nucleus of Cajal (Crawford and Vilis 1993) play a crucial role in the gaze holding system. In addition, the OKN response is highly sensitive to any type of disturbance of the neural integrator (Cheron et al. 1986b).

A change in the preferred vertical direction of gaze may also explain the reversal in vertical vestibulo-ocular reex (VOR) (Clarke et al. 2000) and in vertical OKN (Clément et al. 1986, 1993; Clément 2003) in weightlessness. Indeed, judgements of slope based on the observation of static images also evoke up/down asymmetries of perception (Proftt et al. 1995; Vidal et al. 2006), although not as strong as in the presence of OKN-inducing visual ow. In fact, static gaze and OKN are linked, and so it is perhaps not surprising to see both correlated with the perceptual asymmetry. In accordance with Alexander's Law, the eye velocity of the slow phase increases when the gaze is displaced in the direction of the beating phase of the nystagmus and decreases with gaze in the opposite direction (Lackner and DiZio 2000). An upward displacement of the OKN beating eld in weightlessness would produce both an increase in the downward slow phase velocity and a decrease in the upward slow phase velocity, which would invert the original up-down asymmetry observed in terrestrial condition. Another neural network devoted to the velocity storage mechanism and related to the optokinetic after nystagmus (OKAN) is assumed by the commissural bers linked to the vestibular-prepositus complex of both sides (Godaux and Cheron 1991). This vestibular network is also known to be inuenced by gravity (Dai et al. 1991, 1994) and may inu ence the perception of up and down.

Modication to the control of eye position and move ment in weightlessness provides an attractive hypothesis to explain our experimental observations in weightlessness. The comparison of the attached and free-oating conditions on orbit tell us that this is not, however, the whole story. Vestibular inputs to the CNS were essentially the same in these two conditions. Otoliths are unloaded, whatever the orientation of the head in the local environment, and any rotations or linear accelerations of the head in the free-oating condi tion were of very low amplitude, below the threshold of the vestibular organs. Yet, the asymmetry differed signicantly between the attached and free-oating postures on the rst day of testing in weightlessness. Vestibular drive to eye movement circuitry alone cannot, therefore, fully explain 
the differences in visual perception between ground and weightlessness.

\section{Multisensory cognitive context}

We argue instead for a top-down effect of cognitive context, based on the fusion of multisensory cues as the basis for our observations (cf. Vidal et al. 2006). Humans are thought to maintain an internal representation of the subjective vertical (Mittelstaedt 1983, 1999; Berthoz 1991) that results from a convergence of otolithic cues, somatosensory cues (haptic, proprioception), and visual cues about the environment's vertical (trees, walls...). Due to the multisensory nature of this mechanism on the one hand, and to the ambiguity of otolith and other gravito-inertial signals during motion on the other, this vertical reference frame is not strictly coupled to gravity and may be perturbed during spaceight (Young et al. 1984; Parker et al. 1985). Therefore, one can imagine that in the absence of gravity, this subjective vertical is built upon the remaining cues, to the point that a clear vertical feeling may remain. Indeed, numerous studies have shown that perceptual anisotropies (Appelle 1972; Lipshits and McIntyre 1999; Luyat and Gentaz 2002) rely on a multisensory vertical, rather than on a strict gravitational reference frame.

In our experiments, subjects could rely on visual memory even though external visual cues were excluded from view. When a participant gets ready to perform these experiments, he or she sees the walls and the entire surrounding visual scene. If the subject is aware of the fact that body orientation will not change with respect to this initial view, as on the ground or in the attached position on orbit, the remembered visual scene can serve as a stable reference frame for orientation estimation. In the free-oating condition, subjects could not, however, be assured that the body axis would remain aligned with respect to the remembered visual eld.

The two different conditions used on orbit (attached and free-oating) also differed by the fact that when in a secured seated position subjects could rely on tactile receptors and the stable attachment of the body to the oor of the space station to perceive their body orientation, and hence the orientation of the tunnel's bend, with respect to the stable platform of the ISS. Together with the remembered visual cues, this additional somatosensory information could be enough to maintain a strong sense of verticality, preserving in the attached condition of the perceptual asymmetry. Conversely, subjects in the free-oating condition had no contact with the stable reference frame provided by the station. In this situation, the subject will presumably adopt the body axis as the local vertical and will process pitch and yaw turns accordingly in this local reference frame. Nevertheless, tactile or haptic cues may be critical for the interpretation of ambiguous otolithic signals, even on Earth (Bortolami et al.
2006). Thus, in free-oating, up and down are ambiguous, leading to symmetric overshoot of perceived tunnel angle for pitch up and pitch down tunnels. Being locally "horizontal," one would still expect no overshoot and no asymmetry for left or right yaw with respect to the body axis. A similar, multisensory argument has been applied to explain illusions of being inverted in weightlessness (Simons and Gardner 1963; Graybiel and Kellogg 1967; Lackner 1992), anticipation of the effects of gravity on moving objects even when gravity is not in play (McIntyre et al. 2001; Miller et al. 2008), up/down asymmetries in the perception and interception of moving objects (Senot et al. 2012; Moscatelli and Lacquaniti 2011; Zago et al. 2009; Le Seac'h et al. 2010), and differential tuning of kinematics for upward and downward arm movements (Le Seac'h and McIntyre 2007). Tactile stimulation has even been proposed as a means to combat sensations of spatial disorientation (Van Erp et al. 2006).

It is fascinating to note, therefore, that the asymmetry effect disappeared even in the attached posture at some point between the rst and second time the experiment was performed on orbit. Note that we initially analyzed the rst and last block of trials performed in weightlessness, corresponding to the trials performed with the least and the most amount of practice in each condition, respectively. We reasoned that subjects might quickly adapt responses to weightless conditions with practice. Nevertheless, the results were the same when we considered together all trials from the rst day of testing in weightlessness; that is, the asymme try index was reduced in weightlessness on average in the free-oating condition, but not in the attached condition, and this despite the fact that half of the subjects performed the attached condition rst, and the other half performed the free-oating condition rst.

One might surmise, therefore, that modications to the perception of turn angle required a longer exposure to weightlessness when in the attached posture, since responses in the two conditions differed mainly depending on the test day. But we could not nd evidence that the exposure time to weightlessness, by itself, was the critical factor; indeed, the one subject who performed the tests relatively late during the ight still showed a large asymmetry for his rst testing session even after spending 14 days on orbit. Instead, it appears that the asymmetry disappeared after a certain amount of practice on the task in the attached condition in weightlessness. One possible mechanism for this effect might stem from the high correlation between haptic and vestibular cues that usually occurs in normal gravity. According to recent theories, this correlation would be imbedded in multisensory integration networks, such that the CNS essentially replaces or reconstructs missing gravitational cues with redundant information from other sensory signals (Droulez and Darlot 1989; Pouget et al. 2002; Tagliabue and McIntyre 2011). These associations 
between sensory signals from different modalities are presumably malleable, such that with repeated practice in the absence of up/down vestibular cues, the haptic cues lose signicance, eventually resulting in similar responses between the attached and free-oating conditions overtime. We can not say, however, that the only adaptation is for the haptic cues in the attached condition. Although still not signi cantly different from zero, measurements of asymmetry in

free-oating on the second day of testing (Fig. 4b) showed more inter-subject variability, the average was somewhat higher than on the rst day (though not signicantly), and the asymmetry was no longer signicantly different from pre-ight values. This suggests that some subjects may have adapted toward a return to asymmetric perception of pitch turns even during free-oating, perhaps based on a local ref erence aligned with the body (Miller et al. 2008; Dyde et al. 2006; Le Seac'h et al. 2010). Note that the asymmetry returned immediately on return to Earth, showing that clear, coherent graviceptor cues are sufcient to reinstate the per ceptual asymmetry. Still, it is intriguing to observe that our group of cosmonauts, 6 of whom had previous experience in orbital space ight, manifested a lower level of asymmetry than our control subjects on the ground. The effects of longterm exposure to weightlessness might nevertheless have a permanent effect on this particular percept.

Top-down inuences and affordances

While the multisensory interpretation may explain how the up/down perceptual asymmetry is modulated by the presence or absence of gravity, it does not provide a direct explanation as to why this asymmetry should exist in the rst place. The answer may lie in the analysis of sensory information in the context of what potential actions are afforded by the visual scene. On Earth, humans have developed the capacity to stabilize their posture in the anteroposterior and mediolateral directions in order to overcome gravity constraints and prevent falling (Redfern et al. 2001; Yu et al. 2010). For an upright stance and bipedal locomotion, the risk of falling on any given slope is greater when moving down versus moving upward. The tendency for human subjects to overestimate downward slopes is, therefore, likely to be a manifestation of this perceived danger. The fact that the overestimation increases for dynamic stimuli, implying that the subject is in motion, is coherent with this interpretation. But "falling" only has meaning in a normal gravitational environment for which there is a stable, clearly dened up and down. It is, therefore, possible that subjects confounded "up" and "down" with respect to a body-centered vertical axis (see above) or perceived an equal danger for upward and downward slopes, in the free-oating condition, lead ing to the symmetric overestimation of pitch angle that we observed in both directions (Fig. 3c). In the attached condition, subjects could substitute stable haptic cues for actual graviceptor information and perceived downward versus upward slope accordingly, at least initially. Yaw rotations, however, are benign in terms of postural stability in a normal gravitational eld and thus should be immune to this perceptual distortion when the body axis is aligned with the vertical. Indeed, we saw no asymmetry for yaw stimuli perceived either on the ground (Fig. 3d, e) or in free-oating (Fig. 3f), supporting the notion that in the absence of gravitational or haptic cues, subjects adopt the body axis as the local vertical reference in weightlessness (Le Seac'h et al. 2010). Yaw stimuli did, however, elicit an up/down asymmetry both with respect to gravity and with respect to the body axis when subject was lying on their sides (Vidal et al. 2006). Explaining the asymmetry in terms of affordances in local versus external reference frames argues, therefore, for top-down modulation of the low-level sensory signals that lead to the perception of slope.

Neural pathways, self-motion perception and weightlessness

The distinction between low-level circuits and high-level cognitive function is, in reality, probably not so clear-cut as theoretical discussions of brain function might imply. A more compelling question is that of where the required multisensory processing is carried out. During self-motion, the retina is specically activated by the optic ow (Angelaki and Hess 2005), and these inputs related to the directional velocity of the image on the retina are conducted via the nuclei of the optic tract (NOT) and the nuclei reticular tegmentum pontis (NRTP) up through the vestibular complex and the cerebellum and then forwarded to the vestibular cortical network where the nal perception is probably elabo rated (Kahane et al. 2003; Indovina et al. 2005; Maffei et al. 2010). There are thus many loci likely to be affected by weightlessness.

The convergence in the vestibular nuclei of the inputs related to head movement and those related to the moving image on the retina may be considered as a rst reasonable site inuenced by weightlessness. On Earth, if the head is xed in space, the output from this neural site is able to pro duce the sensation of a downward pitch only on the basis of upward optical ux. But the absence of head movement does not mean that the information from the vestibular afferents is not implicated in the downward pitch sensation. When the head is horizontal, the resting discharge pattern of the otolith organs of the inner ear provides a static reference; deviations from that baseline are integrated in the convergent vestibular network, giving rise to perception of body movement. In weightlessness, the otolith organs are unloaded, and the static otolith-spinal and otolith-oculomotor inuences are missing. The loss of these anchoring cues 
might thus explain the alteration in the perception of upward and downward pitch.

\section{Conclusions}

With this experiment, we demonstrate that the asymmetrical perception of upward and downward pitch is suppressed in weightlessness. However, this perceptual anisotropy is initially preserved in the "attached" condition and disappeared only after practice in weightlessness. The reported effects of weightlessness on the early processing stages (vestibular and optokinetics) that underlie the perception of self-motion may, therefore, be partially overcome by haptic cues, reect ing the fusion of multisensory cues and top-down inuences on visual perception, based on the affordances of the local environment.

Acknowledgments This work was funded by the Belgian Federal Science Policy Ofce, the European Space Agency (AO-2004, 118), the Belgian National Fund for Scientic Research (F.N.R.S.), the Research Fund of the University of Brussels (U.L.B.), Russian research grant RFBR 11-04-01068 and the French space agency CNES (Centre National des Etudes Spatiales). The authors would like to thank the cosmonauts who participated in this experiment, and the personnel at ESA, CNES, Star City, and Tsoup who made this space experiment possible, especially D. Chaput, E. Lorigny, and V. Grachev. We thank P. Demaret and E. Hortmanns for expert technical assistance.

\section{References}

Angelaki DE, Hess BJ (2005) Self-motion-induced eye movements: effects on visual acuity and navigation. Nat Rev Neurosci 6(12):966-976

Appelle S (1972) Perception and discrimination as a function of stimulus orientation: the "oblique effect" in man and animals. Psychol Bull 78(4):266-278

Berthoz A (1991) In brain and space, reference frames for the perception and control of movement. Oxford University Press, Oxford, pp 82-111

Bortolami SB, Rocca S, Daros S, DiZio P, Lackner JR (2006) Mechanisms of human static spatial orientation. Exp Brain Res 173:374-388

Cheron G, Godaux E (1987) Disabling of the oculomotor neural integrator by microinjections of kainic acid in the prepositus vestibular nuclear complex of the cat. J Physiol (Lond) 394:267-290

Cheron G, Godaux E, Laune J-M, Vanderkelen B (1986a) Lesions in the cat prepositus complex: effects on the vestibulo-ocular reex and saccades. J Physiol (Lond) 372:75-94

Cheron G, Godaux E, Vanderkelen G (1986b) Lesions in the cat prepositus complex: effects on the optokinetic system. J Physiol (Lond) 372:95-111

Cheron G, Leroy A, De Saedeleer C, Bengoetxea A, Lipshits M, Cebolla A, Servais L, Dan B, Berthoz A, McIntyre J (2006) Effect of gravity on human spontaneous $10-\mathrm{Hz}$ electroencephalographic oscillations during the arrest reaction. Brain Res 1121(1):104-116

Clarke AH, Grigull J, Mueller R, Scherer H (2000) The three-dimensional vestibulo-ocular reex during prolonged microgravity. Exp Brain Res 134(3):322-334
Clément G (2003) A review of the effects of space ight on the asym metry of vertical optokinetic and vestibulo-ocular reexes. J Vestib Res 13(4-6):255-263

Clément G, Lathan CE (1991) Effects of static tilt about the roll axis on horizontal and vertical optokinetic nystagmus and optokinetic after-nystagmus in humans. Exp Brain Res 84(2):335-341

Clément G, Reschke M (2008) Neuroscience in space. Springer, New York

Clément G, Vieville T, Lestienne F, Berthoz A (1986) Modications of gain asymmetry and beating eld of vertical optokinetic nystag mus in microgravity. Neurosci Lett 63(3):271-274

Clément G, Popov KE, Berthoz A (1993) Effects of prolonged weightlessness on human horizontal and vertical optokinetic nystagmus and optokinetic after-nystagmus. Exp Brain Res 94:456-462

Crawford JD, Vilis T (1993) Modularity and parallel processing in the oculomotor integrator. Exp Brain Res 96:443-456

Dai MJ, Raphan T, Cohen B (1991) Spatial orientation of the vestibular system: dependence of optokinetic after-nystagmus on gravity. J Neurophysiol 66(4):1422-1439

Dai M, McGarvie L, Kozlovskaya I, Raphan T, Cohen B (1994) Effects of spaceight on ocular counterrolling and the spatial orientation of the vestibular system. Exp Brain Res 102(1):45-56

Droulez J, Darlot C (1989) The geometric and dynamic implications of the coherence constraints in three dimensional sensorimotor interactions. In: Jeannerod M (ed) Attention and performance XIII. Erlbaum, London, pp 495-526

Dyde RT, Jenkin MR, Harris LR (2006) The subjective visual vertical and the perceptual upright. Exp Brain Res 173:612-622

Fuglesang C, Narici L, Picozza P, Sannita WG (2006) Phosphenes in low earth orbit: survey responses from 59 astronauts. Aviat Space Environ Med 77(4):449-452

Gizzi M, Raphan T, Rudolph S, Cohen B (1994) Orientation of human optokinetic nystagmus to gravity: a model-based approach. Exp Brain Res 99(2):347-360

Glasauer S, Mittelstaedt H (1998) Perception of spatial orientation in microgravity. Brain Res Rev 28:185-193

Godaux E, Cheron G (1991) Effects of severance of the vestibular commissural pathway on the neural integrator of the oculomotor system in the cat. Oculomot Control Cogn Process (Schmid and Zambarbieri eds) 2:39-62

Graybiel A, Kellogg RS (1967) Inversion illusion in parabolic ight: its probable dependence on otolith function. Aerosp Med 38(11):1099-1103

Igarashi M, Himi T, Kulecz WB, Patel S, The role of saccular afferents in vertical optokinetic nystagmus in primates (1987) A study in relation to optokinetic nystagmus in microgravity. Arch Otorhinolaryngol 244(3):143-146

Indovina I, Maffei V, Bosco G, Zago M, Macaluso E, Lacquaniti F (2005) Representation of visual gravitational motion in the human vestibular cortex. Science 308:416-419

Kahane P, Hoffmann D, Minotti L, Berthoz A (2003) Reappraisal of the human vestibular cortex by cortical electrical stimulation study. Ann Neurol 54:615-624

Kornilova LN, Kreĭdich IuV, Tarasov IK, Iakovleva IIa (1983) Optokinetic nystagmus and optokinetic resistance of cosmonauts in preight and postight periods. Kosm Biol Aviakosm Med 17(4):12-15

Lackner JR (1992) Sense of body position in parabolic ight. Ann N Y Acad Sci 656:329-339

Lackner JR, DiZio P (2000) Human orientation and movement control in weightless and articial gravity environments. Exp Brain Res 130(1):2-26

Le Seac'h AB, McIntyre J (2007) Multimodal reference frame for the planning of vertical arms movements. Neurosci Lett 423: 211-215 
Le Seac'h AB, Senot P, McIntyre J (2010) Egocentric and allocentric reference frames for catching a falling object. Exp Brain Res 201(4):653-662

Lipshits M, McIntyre J (1999) Gravity affects the preferred vertical and horizontal in visual perception of orientation. NeuroReport 10:1085-1089

Lipshits M, Bengoetxea A, Cheron G, McIntyre J (2005) Two reference frames for visual perception in two gravity conditions. Perception 34:545-555

Luyat M, Gentaz E (2002) Body tilt effect on the reproduction of orientations: studies on the visual oblique effect and subjective orientations. J Exp Psychol Hum Percept Perform 28:1002-1011

Maffei V, Macaluso E, Indovina I, Orban G, Lacquaniti F (2010) Processing of targets in smooth or apparent motion along the vertical in the human brain: an fMRI study. J Neurophysiol 103(1):360-370

Major G, Baker R, Aksay E, Seung HS, Tank DW (2004) Plasticity and tuning of the time course of analog persistent ring in a neural integrator. Proc Natl Acad Sci USA 101(20):7745-7750

McIntyre J, Zago M, Berthoz A, Lacquaniti FR (2001) Does the brain model Newton's laws? Nat Neurosci 4:693-694

Miller WL, Maffei V, Bosco G, Iosa M, Zago M, Macaluso E, Lacquaniti F (2008) Vestibular nuclei and cerebellum put visual gravitational motion in context. J Neurophysiol 99(4):1969-1982

Mittelstaedt H (1983) A new solution to the problem of the subjective vertical. Naturwissenschaften 70:272-281

Mittelstaedt H (1999) The role of the otoliths in perception of the vertical and in path integration. Ann N Y Acad Sci 871:334-344

Moscatelli A, Lacquaniti F (2011) The weight of time: gravitational force enhances discrimination of visual motion duration. J Vis 11(4):5, 1-17

NASA's Extended Duration Orbiter Medical Project (EDOMP), Final Report, 1989-1995, NASA/SP-1999-534

Parker DE, Reschke MF, Arrott AP, Homick JL, Lichtenberg BK (1985) Otolith tilt-translation reinterpretation following prolonged weightlessness: implications for preight training. Aviat Space Environ Med 56(6):601-606

Popov V, Boyko N (1967) Vision in space travel. Aviatsiya i Kosmonaut 3:73-76

Pouget A, Deneve S, Duhamel JR (2002) A computational perspective on the neural basis of multisensory spatial representations. Nat Rev Neurosci 3:741-747

Pozzo T, Papaxanthis C, Stapley P, Berthoz A (1998) The sensorimotor and cognitive integration of gravity. Brain Res Brain Res Rev 28(1-2):92-101

Proftt DR, Bhalla M, Gossweiler R, Midgett J (1995) Perceiving geo graphical slant. Psychon Bull Rev 2:409-428
Redfern MS, Yardley L, Bronstein AM (2001) Visual inuences on balance. J Anxiety Disord 15(1-2):81-94

Robinson DA (1989) Integrating with neurons. Ann Rev Neurosci $12: 33-45$

Sciutti A, Demougeot L, Berret B, Toma S, Sandini G, Papaxanthis C, Pozzo T, Sciutti A, Demougeot L, Berret B, Toma S, Sandini G, Papaxanthis C, Pozzo T (2012) Visual gravity inuences arm movement planning. J Neurophysiol 107(12):3433-3445

Senot P, Zago M, Le Seac'h A, Zaoui M, Berthoz A, Lacquaniti F, McIntyre JJ (2012) When up is down in $0 \mathrm{~g}$ : how gravity sensing affects the timing of interceptive actions. Neurosci 32(6):1969-1973

Simons JC, Gardner MS (1963) Weightless man: a survey o f sensations and performance while free-oating. Wright-Patterson Air Force Base OH; (USAF Tech. Rep. AMRL-TDR-62-114)

Tagliabue M, McIntyre J (2011) Necessity is the mother of invention: reconstructing missing sensory information in multiple, concurrent reference frames for eye-hand coordination. J Neurosci 31:1397-1409

Task HL, Genco LV (1987) Effects of short-term space ight on sev eral visual functions. In: Bungo MW, Bagian TM, Bowman MA, Levitan BM (eds) Results of the bye sciences DSOs conducted aboard the space shuttle 1981-1986. NASA Technical Memorandum 58280, p 173

Van Erp JB, Groen EL, Bos JE, van Veen HA (2006) A tactile cockpit instrument supports the control of self-motion during spatial disorientation. Hum Factors 48(2):219-228

Vidal M, Bülthoff HE (2009) Storing upright turns: how visual and vestibular cues interact during the encoding and recalling process. Exp Brain Res 200(1):37-49

Vidal M, Lipshits M, McIntyre J, Berthoz A (2003) Gravity and spatial orientation in virtual 3D-mazes. J Vestib Res 13:273-286

Vidal M, Amorim MA, McIntyre J, Berthoz A (2006) The perception of visually presented yaw and pitch turns: assessing the contribution of motion, static, and cognitive cues. Percept Psychophys 68:1338-1350

Young LR, Oman CM, Watt DG, Money KE, Lichtenberg BK (1984) Spatial orientation in weightlessness and readaptation to earth's gravity. Science 225(4658):205-208

Yu Y, Yank JR, Katsumata Y, Villard S, Kennedy RS, Stoffregen TA (2010) Visual vigilance performance and standing posture at sea. Aviat Space Environ Med 81(4):375-382

Zago M, McIntyre J, Senot P, Lacquaniti F (2009) Visuo-motor coordination and internal models for object interception. Exp Brain Res 192(4):571-604 